\title{
Synthesis of Tetra(imidazoil-4(5)-yl)pyrazine and Tetrapyrazilpyrazine
}

\author{
Carolina B. P. Ligiéro; Luis G. T. A. Duarte; Paulo C. M. L. Miranda
}

Instituto de Química, Universidade Estadual de Campinas, SP

*e-mail corresponding author: miranda@iqm.unicamp.br

Keywords: Supramolecular chemistry, benzoin condensation, polyheteroaromatic ligands

\section{INTRODUCTION}

$\mathrm{N}$-heterocyclic bridging ligands are able of forming complexes with metals allowing the construction of functional supramolecular systems.

We present the synthesis of two new building blocks designed for the construction of tridimensional arrays: 2,3,5,6-tetra(pyrazin-2-yl)pyrazine (1) and 2,3,4,6-tetra(imidazol-4(5)-yl)pyrazine (2).

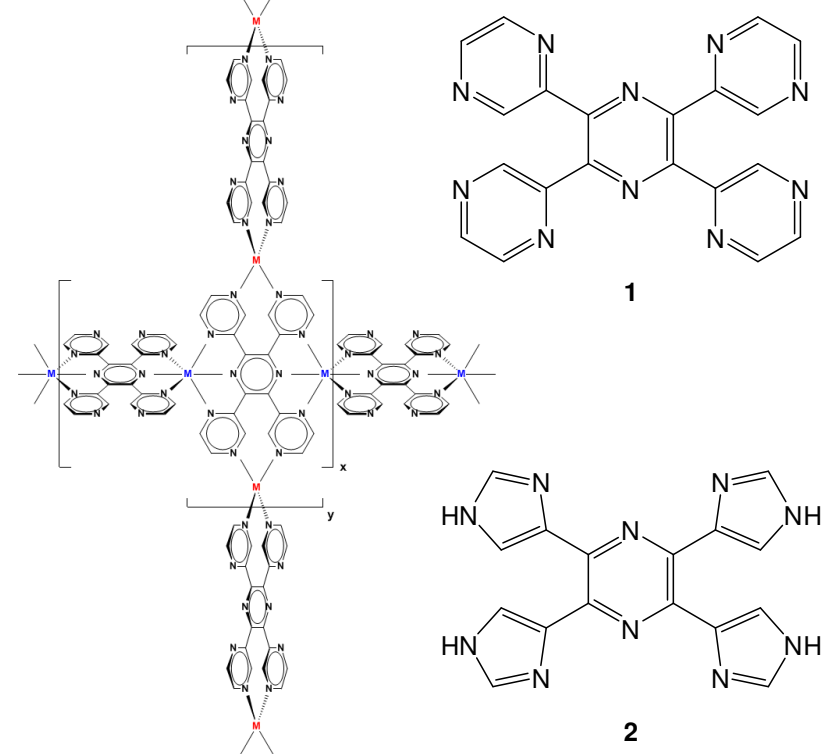

Figure 1. Tetrapyrazilpyrazine (1) and tetra(imidazol-4(5)yl)pyrazine (2).

\section{RESULTS AND DISCUSSION}

Tetrapyrazilpyrazine was prepared in a four step route from pyrazinecarboxilic acid with $15 \%$ of global yield (Scheme 1) from benzoin coupling strategy. ${ }^{1}$ Tetra(imidazol-4(5)yl)pyrazine was also synthesized by benzoin condensation strategy, but in this case we used 4(5)-imidazolecarbaldehyde as starting material (Scheme 2). Protection with sulfamoyl group was critical to activate the aldehyde carbonyl in the benzoin condensation. Many other protecting groups failed in performing this reaction. With BOC, acetyl and tosyl as protecting groups ANRORC degradation products prevail over benzoin coupling.<smiles>O=C(O)c1cncc(-c2cnccn2)c1</smiles>

a) $\mathrm{MeOH}, \mathrm{H}_{2} \mathrm{SO}_{4}$ (cat), $72 \mathrm{~h}$, rt., $93 \%$ b) $\mathrm{LiAlH}_{4}, \mathrm{THF}, 1.5 \mathrm{~h},-82^{\circ} \mathrm{C}$, $63 \%$ c) $\mathrm{KCN}$ (cat), water, rt., 1 h, $73 \%$ d) $\mathrm{NH}_{4} \mathrm{OAc}$, DMF, $80^{\circ} \mathrm{C}$, $36 \%$.

Scheme 1. Synthesis of tetrapyrazilpyrazine.<smiles>O=Cc1c[nH]cn1</smiles><smiles>CN(C)S(=O)(=O)n1cnc(C=O)c1</smiles><smiles>CC(C)(C)Sn1cnc(C(O)C(=O)c2cn(S(C)(=O)=O)cn2)c1</smiles>

a) $\mathrm{NaH}, \mathrm{DMF}, \mathrm{rt}, 1.5 \mathrm{~h}$ and then DMASCl, rt, $24 \mathrm{~h}, 68 \%$ b) $3-$ (benzyl)-5-(2-hydroxyethyl)-4-methylthiazolium chloride, EtOH, $50^{\circ} \mathrm{C}, 30 \mathrm{~min}, 93 \%$ c) $\mathrm{NH}_{4} \mathrm{OAc}$, pyridine, $100^{\circ} \mathrm{C}, 2 \mathrm{~h}, 20 \%$ d) $\mathrm{HCl}$ $6 \mathrm{M}, 100^{\circ} \mathrm{C}, 64 \%$.

Scheme 2. Synthesis of tetra(imidazol-4(5)yl)pyrazine.

\section{CONCLUSION}

Two new ligands for supramolecular assembling were successfully synthesized from simple heteroaromatic compouds in few steps.

\section{ACKNOWLEDGEMENTS}

CNPQ, CAPES, FAPESP, INCT-INOMAT.

\section{REFERENCES}

1 Torres, S. F.; Hutchison, G. R.; Stolzberg, L. J.; Abruña, H. D. J. Am. Chem. Soc. 2006, 128, 1513.

${ }^{2}$ Ligiero, C. B. P.; Visentin L. C.; Giacomini, R; Filgueiras, C. A. L.; Miranda, P. C. M. L. Tetrahedron Lett. 2009, 50, 4030. 